equatorium is a rare and complex instrument, and is not easy for non-astronomers to understand. The example illustrated, one of only a handful known, is in the collection of the National Museums and Galleries at Merseyside, in the United Kingdom. A cropped version is used as the colour illustration on the front cover, but very few readers would recognize it. A better, and perhaps more symbolic, choice might have been the Powell and Lealand microscope of 1846. Just 60 years later, Charles Rolls and Henry Royce started making cars, in the same limited quantity but to the same exacting standards.

One of my sons is presently reading for his MSc. I considered giving him my review copy of this encyclopedia but, on second thoughts, I shall make good use of it myself. Arthur Middleton, a dealer in the history of science, is at 12 New Row, Covent Garden, London WC2N 4LF, UK.

\section{Darwin's fixed \\ course}

\section{Evolution: Society, Science and the Universe \\ edited by A. C. Fabian \\ Cambridge University Press: 1998. Pp. 179.

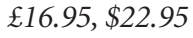 \\ Mark Pagel}

The irony of the modern tendency to use the word 'evolution' as synonymous with natural selection is that, like the traits of many species, it may reveal something of its ancestry. In Darwin's time, 'evolve' meant to unfold, roll out or unfurl, suggesting a fixed course. He anxiously wished to avoid this meaning for his new-found 'natural selection', and so it may not be surprising that he used 'evolve' only once in the first edition of The Origin of Species, and even then he waited until the very end, to the last word of the book. And yet, some of the products of natural selection are so exquisite as to suggest something preordained, and 'evolve' in its old denotative sense finds its way back into our discourse.

Meanings change, however. The contemporary scientific concept of evolution as simply 'to change over time' is ever more widely applied, and not just to refer to living things. A. C. Fabian's delightful new edited volume is a case in point. Fabian invited eight wellknown "communicators from separate disciplines" to discuss evolution in the successful Darwin College lecture series at the University of Cambridge. The result is an eclectic collection that can fail to interest only the modern philistine.

The possibility that forms of natural selection lurk as 'invisible hands' in areas well removed from biology does not always settle easily in the mind of the essayist. In her

\section{Face value}

The arrangement of features in the human face largely reflects sensory, dietary and linguistic considerations, so all faces look more or less the same. This means that minor variations can effectively convey social signals and information about identity. This is why natural facial disfigurements have such a profound effect on people's lives. It also explains the disturbing effect of artistic violations of the face, such as this striking painting, Le Viol (The Rape) by René Magritte. Or so argue Vicki Bruce and Andy Young in In the Eye of the Beholder: The Science of Face Perception (Oxford

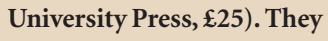
bring together science and art to explain the importance of the face, how we extract the information it contains, and reveal what it all means.

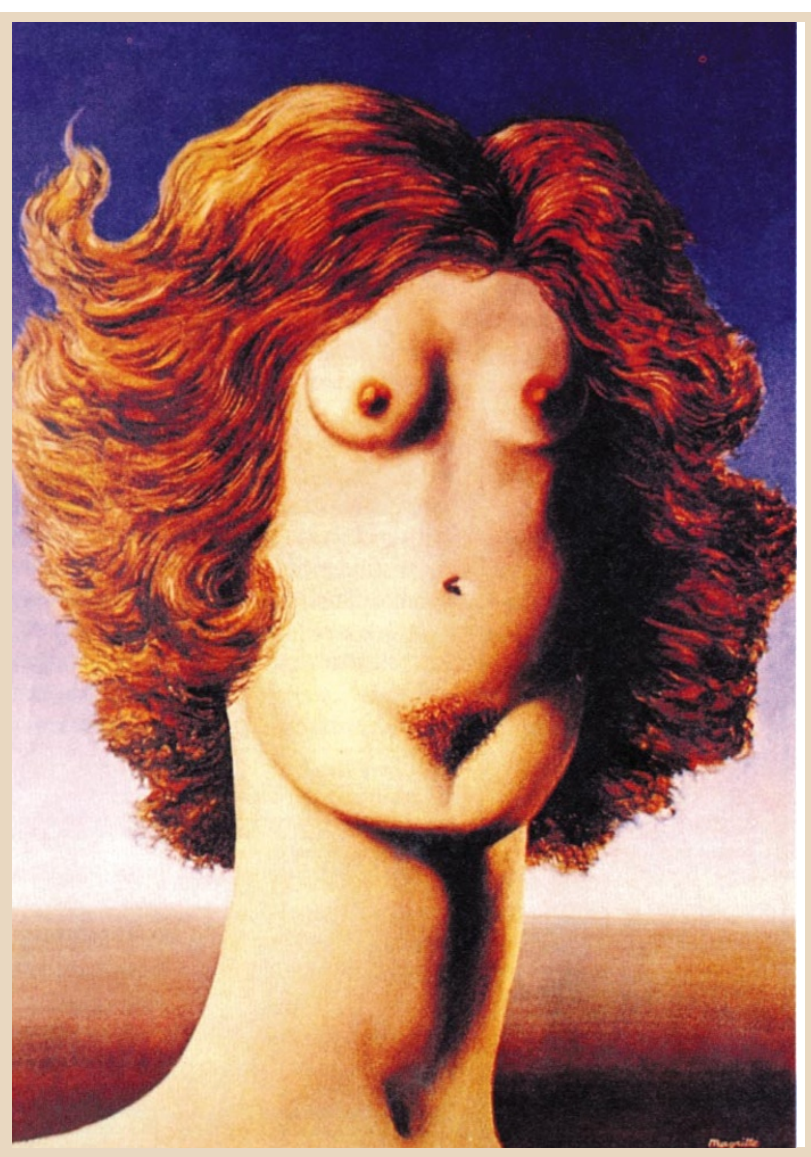

chapter, Gillian Beer allows that readers and novels have evolved. She is less willing to countenance the view that works outside the literary canon, although less 'fit', are somehow less worth reading. The architect Richard Rogers argues passionately that London has been in steady decline since the mid-1980s when the government removed its central planning authority. Rogers and Beer are making a point well known to biologists: that which emerges from the unsentimental struggle for survival is the fittest, but not necessarily the most desirable.

Biologists wishing to understand human civilization from a darwinian perspective confront a dilemma, avers Tim Ingold. All human cultural evolution has taken place against the same biological background: Fred Flintstone, had he been born later, might have been Shakespeare or Einstein. This, Ingold says, forces biologists to adopt a 'progressive' view of history as the "unfolding of preevolved potentials", with 'primitive' huntergatherers at the bottom and Western scientists at the top: a "fundamentally teleological view!” Ingold exclaims triumphantly.

But cultural evolution is not a one-way street: cultures adopt more 'primitive' subsistence practices when it is advantageous to do so and, as Jared Diamond's informative chapter summarizes, they lose as well as acquire technologies. He records how Tasmanian islanders lost the ability to fish and even to start fires following their geographical separation from mainland Australians.
Ingold's position shows how difficult it can be to translate the arguments and assumptions of one field to another.

Still, evolutionary processes may unfold in more subtle ways than is realized. The astronomer Martin Rees reluctantly ponders whether our Universe could have been much different from what we see, given the laws of physics that emerged with the Big Bang. A similar question worries biologists: are the forms we see now (such as kangaroos, toadstools, fruitflies and sequoias) mere accidents, or if we were to re-run evolution would similar (if not the same) kinds of organisms emerge? Intriguingly, evidence is pointing towards the latter view, confounding many a sceptic. And what of cities and even novels? The future may not be preordained but its broad outlines might be.

The long march of evolution now includes spin-offs into other fields; E. O. Wilson sees this as a process of 'conciliance'. This was not lost on Fabian, who, having brought his speakers to Cambridge, produced this fine volume. With contributors ranging from those discussed here to Freeman Dyson, Lewis Wolpert and Stephen Jay Gould, Fabian's book reaffirms my view that the best researchers and scientists often make the best science writers: no exaggerated claims, no mysterious theories of everything, no yin and yang. Just a joy to read. Mark Pagel is in the Department of Zoology, University of Oxford, South Parks Road, Oxford OX1 3PS, UK. 